\title{
Two Gilbert-Varshamov-type existential bounds for asymmetric quantum error-correcting codes
}

\author{
Ryutaroh Matsumoto ${ }^{1}$
}

Received: 11 May 2017 / Accepted: 12 October 2017 / Published online: 23 October 2017

(C) The Author(s) 2017. This article is an open access publication

\begin{abstract}
In this note, we report two versions of Gilbert-Varshamov-type existential bounds for asymmetric quantum error-correcting codes.
\end{abstract}

Keywords Asymmetric error · Quantum error correction · Gilbert-Varshamov bound

Mathematics Subject Classification 94B65

\section{Introduction}

Quantum error-correcting codes (QECCs) are important for construction of quantum computers, as the fault-tolerant quantum computation is based on QECC [13]. There are two kinds of errors in quantum information: One is called a bit error, and the other is called a phase error. Steane [16] first studied the asymmetry between probabilities of the bit and the phase errors, and he also considered QECC for asymmetric quantum errors, which are called asymmetric quantum error-correcting codes (AQECC). Research on AQECC has become very active recently; see $[7,9,16]$ and the references therein.

On the other hand, in the study of error-correcting codes, it is important to know the optimal performance of codes. For classical error-correcting codes, the GilbertVarshamov (GV) bound [11] is a sufficient condition for existence of codes whose

Ryutaroh Matsumoto

ryutaroh@rmatsumoto.org

http://www.rmatsumoto.org/research.html

1 Department of Communication and Computer Engineering, Nagoya University, Nagoya 464-8603, Japan 
parameters satisfies the GV bound. By the GV bound, one can know that the optimal performance of classical codes is at least as good as the GV bound.

For QECC, Ekert and Macchiavello obtained a GV-type existential bound for general QECCs. An important subclass of general QECCs is the stabilizer codes [2,3,8], as they enable efficient encoding and decoding. Calderbank et al. [2] obtained a GV-type existential bound for the stabilizer QECCs. After that, Feng and Ma [6] and Jin and Xing [10] obtained improved versions of GV-type bounds for the stabilizer QECCs.

The Calderbank-Shor-Steane (CSS) QECCs [4,15] are an important subclass of the stabilizer QECCs, as the CSS codes enable more efficient implementation of the fault-tolerant quantum computation than the stabilizer codes.

Those existential bounds $[2,4-6,10]$ did not consider the asymmetric quantum errors, while the asymmetry in quantum errors is important in practice [14]. As far as the author knows, nobody has reported existential bounds for the stabilizer or the CSS QECC for asymmetric quantum errors. In this note, we report such ones. Our proof arguments are similar to ones in $[2,4]$.

\section{A GV-type existential bound for the CSS codes}

An $\left[\left[n, k, d_{x}, d_{z}\right]\right]_{q}$ QECC encodes $k q$-ary qudits into $n q$-ary qudits and detects up to $d_{x}$ bit errors and up to $d_{z}$ phase errors. It is known $[1,3]$ that a nested classical code $C_{2} \subset C_{1} \subset \mathbf{F}_{q}^{n}$ with dimensions $k_{2}$ and $k_{1}$ can construct an $\left[\left[n, \operatorname{dim} C_{1}-\operatorname{dim} C_{2}\right]\right]_{q}$ CSS code, where $\mathbf{F}_{q}$ is a finite field with $q$ elements. A quantum error can be expressed as a pair $\left(\mathbf{e}_{x}, \mathbf{e}_{z}\right)$, where $\mathbf{e}_{x} \in \mathbf{F}_{q}^{n}$ corresponds to the bit error component of a quantum error and $\mathbf{e}_{x} \in \mathbf{F}_{q}^{n}$ does to the phase error component.

Let $\mathrm{GL}_{n}\left(\mathbf{F}_{q}\right)$ be the group of $n \times n$ invertible matrices over $\mathbf{F}_{q}$. Let $B_{n}=\left\{\left(C_{1}\right.\right.$, $\left.\left.C_{2}\right) \mid C_{2} \subset C_{1} \subset \mathbf{F}_{q}^{n}, \operatorname{dim} C_{1}=k_{1}, \operatorname{dim} C_{2}=k_{2}\right\}$. For a nonzero vector $\mathbf{e} \in \mathbf{F}_{q}^{n}$, let $B_{n, x}(\mathbf{e})$ (resp. $\left.B_{n, z}(\mathbf{e})\right)$ be the set of nested code pairs that cannot detect $\mathbf{e}$ as a bit error (resp. a phase error), that is, $B_{n, x}(\mathbf{e})=\left\{\left(C_{1}, C_{2}\right) \in B_{n} \mid \mathbf{e} \in C_{1} \backslash C_{2}\right\}$ (resp. $\left.B_{n, z}(\mathbf{e})=\left\{\left(C_{1}, C_{2}\right) \in B_{n} \mid \mathbf{e} \in C_{2}^{\perp} \backslash C_{1}^{\perp}\right\}\right)$, where $C_{1}^{\perp}$ is the dual code of $C_{1}$ with respect to the standard inner product.

Lemma 1 For nonzero e, we have

$$
\begin{aligned}
& \sharp B_{n, x}(\mathbf{e})=\frac{q^{k_{1}}-q^{k_{2}}}{q^{n}-1} \sharp B_{n}, \\
& \sharp B_{n, z}(\mathbf{e})=\frac{q^{n-k_{2}}-q^{n-k_{1}}}{q^{n}-1} \sharp B_{n} .
\end{aligned}
$$

Proof As each pair $C_{2} \subset C_{1}$ has $\sharp C_{1} \backslash C_{2}=q^{k_{1}}-q^{k_{2}}$ undetectable errors, we have

$$
\frac{\sum_{\mathbf{0} \neq \mathbf{e} \in \mathbf{F}_{q}^{n}} \sharp B_{n, x}(\mathbf{e})}{\sharp B_{n}}=q^{k_{1}}-q^{k_{2}} .
$$


For nonzero $\mathbf{e}_{1}, \mathbf{e}_{2} \in \mathbf{F}_{q}^{n}$, we claim $\sharp B_{n, x}\left(\mathbf{e}_{1}\right)=\sharp B_{n, x}(\mathbf{e})_{2}$. Assuming the claim, we have

$$
\sum_{\mathbf{0} \neq \mathbf{e} \in \mathbf{F}_{q}^{n}} \sharp B_{n, x}(\mathbf{e})=\left(q^{n}-1\right) \sharp B_{n, x}(\mathbf{e}) .
$$

Combining these two equalities, we have

$$
\sharp B_{n, x}(\mathbf{e})=\frac{q^{k_{1}}-q^{k_{2}}}{q^{n}-1} \sharp B_{n} .
$$

We finish the proof by proving the claim. Let $\mathbf{e}_{1}, \mathbf{e}_{2}$ be nonzero vectors. We have

$$
\begin{aligned}
\sharp B_{n, x}\left(\mathbf{e}_{1}\right) & =\sharp\left\{\left(C_{1}, C_{2}\right) \in B_{n} \mid \mathbf{e}_{1} \in C_{1} \backslash C_{2}\right\} \\
& =\sharp\left\{\left(\tau C_{1}, \tau C_{2}\right) \mid \tau \in \mathrm{GL}_{n}\left(\mathbf{F}_{q}\right), \mathbf{e}_{1} \in C_{1} \backslash C_{2}\right\} \\
& =\sharp\left\{\left(\tau C_{1}, \tau C_{2}\right) \mid \tau \in \mathrm{GL}_{n}\left(\mathbf{F}_{q}\right), \tau^{\prime} \mathbf{e}_{1} \in C_{1} \backslash C_{2}\right\} \\
& =\sharp\left\{\left(C_{1}, C_{2}\right) \in B_{n} \mid \tau^{\prime} \mathbf{e}_{1} \in C_{1} \backslash C_{2}\right\} \\
& =\sharp B_{n, x}\left(\tau^{\prime} \mathbf{e}_{1}\right),
\end{aligned}
$$

where $\tau^{\prime} \in \mathrm{GL}_{n}\left(\mathbf{F}_{q}\right)$ such that $\tau^{\prime} \mathbf{e}_{1}=\mathbf{e}_{2}$.

For phase errors, we can make a similar argument with $C_{2}^{\perp} \supset C_{1}^{\perp}$.

Theorem 2 Let $n, k_{1}, k_{2}, d_{x}$ and $d_{z}$ be positive integers such that

$$
\frac{q^{k_{1}}-q^{k_{2}}}{q^{n}-1} \sum_{i=1}^{d_{x}-1}\left(\begin{array}{l}
n \\
i
\end{array}\right)(q-1)^{i}+\frac{q^{n-k_{2}}-q^{n-k_{1}}}{q^{n}-1} \sum_{i=1}^{d_{z}-1}\left(\begin{array}{l}
n \\
i
\end{array}\right)(q-1)^{i}<1,
$$

then an $\left[\left[n, k_{1}-k_{2}, d_{x}, d_{z}\right]\right]_{q}$ CSS QECC exists.

Proof Recall that each quantum error can be expressed by its bit error component $\mathbf{e}_{x} \in \mathbf{F}_{q}^{n}$ and its phase error component $\mathbf{e}_{z} \in \mathbf{F}_{q}^{n}$. The bit error component $\mathbf{e}_{x}$ cannot be detected by codes in $B_{n, x}\left(\mathbf{e}_{x}\right)$, and the phase error component $\mathbf{e}_{z}$ cannot be detected by codes in $B_{n, z}\left(\mathbf{e}_{z}\right)$. The detectabilities of the bit errors and the phase errors are independent of each other. Therefore, if Eq. (1) holds, then there exists at least one $\left(C_{1}, C_{2}\right) \in B_{n}$ that can detect all the bit errors with weight up to $d_{x}-1$ and all the phase errors with weight up to $d_{z}-1$, which implies it is an $\left[\left[n, k_{1}-k_{2}, d_{x}, d_{z}\right]\right]_{q}$ quantum code.

Classical coding theorists often have interest in asymptotic versions of GV-type existential bounds [11]. They are stated in terms of information rate and relative distance of classical error-correcting codes. In the classical error correction, information rate is the ratio of the number of information symbols to the code length, and relative distance is the ratio of the minimum distance to the code length.

We can also derive an asymptotic version of Theorem 2. For an $\left[\left[n, k, d_{x}, d_{z}\right]\right]_{q}$ AQECC, we may define the relative distance $\delta_{x}$ for bit errors as $d_{x} / n$ and the relative 
distance $\delta_{z}$ for bit errors as $d_{z} / n$. The information rate of an $[[n, k]]_{q}$ QECC is defined as $k / n[13]$.

Recall [11] that for $0 \leq \delta \leq 1-1 / q$ we have

$$
\sum_{i=1}^{\lfloor n \delta\rfloor}\left(\begin{array}{l}
n \\
i
\end{array}\right)(q-1)^{i} \leq q^{n h_{q}(\delta)},
$$

where $h_{q}(\delta)=\delta \log _{q}(q-1)-\delta \log _{q} \delta-(1-\delta) \log _{q}(1-\delta)$.

Corollary 3 Let $\delta_{x}$ and $\delta_{z}$ be real numbers such that $0 \leq \delta_{x} \leq 1-1 / q$ and $0 \leq \delta_{z} \leq$ $1-1 / q$. If

$$
\begin{aligned}
h_{q}\left(\delta_{x}\right) & <1-R_{1}, \\
h_{q}\left(\delta_{z}\right) & <R_{2}, \text { and } \\
0 & \leq R_{1}-R_{2},
\end{aligned}
$$

then, for sufficiently large $n$, there exists an $\left[\left[n,\left\lfloor n R_{1}\right\rfloor-\left\lceil n R_{2}\right\rceil,\left\lfloor n \delta_{x}\right\rfloor,\left\lfloor n \delta_{z}\right\rfloor\right]\right]_{q} C S S$ QECC exists.

In Corollary $3, R_{1}$ is the information rate of classical ECC $C_{1}$, and $R_{2}$ is the information rate of classical ECC $C_{2}$. The corresponding quantum CSS code has information rate $R_{1}-R_{2}$, relative distance $\delta_{x}$ for bit errors, and relative distance $\delta_{z}$ for phase errors.

Proof Assume that Eq. (3) holds. Then for sufficiently large $n$, we have

$$
\begin{aligned}
& n h_{q}\left(\delta_{x}\right)<n-n R_{1} \\
& \Rightarrow q^{n h_{q}\left(\delta_{x}\right)}<(1 / 2) \frac{q^{n}}{q^{n R_{1}}} \\
& \Rightarrow \frac{q^{n R_{1}}}{q^{n}} q^{n h_{q}\left(\delta_{x}\right)}<1 / 2 \\
& \Rightarrow \frac{q^{\left\lfloor n R_{1}\right\rfloor}-q^{\left\lceil n R_{2}\right\rceil}}{q^{n}-1} \sum_{i=1}^{\left\lfloor n \delta_{x}\right\rfloor-1}\left(\begin{array}{l}
n \\
i
\end{array}\right)(q-1)^{i}<1 / 2 .
\end{aligned}
$$

Similarly, for sufficiently large $n$ Eq. (4) implies

$$
\begin{aligned}
& n h_{q}\left(\delta_{z}\right)<n R_{2} \\
& \Rightarrow q^{n h_{q}\left(\delta_{z}\right)}<(1 / 2) \frac{q^{n}}{q^{n\left(1-R_{2}\right)}} \\
& \Rightarrow \frac{q^{n\left(1-R_{2}\right)}}{q^{n}} q^{n h_{q}\left(\delta_{z}\right)}<1 / 2 \\
& \Rightarrow \frac{q^{n-\left\lceil n R_{2}\right\rceil}-q^{n-\left\lfloor n R_{1}\right\rfloor}}{q^{n}-1} \sum_{i=1}^{\left\lfloor n \delta_{z}\right\rfloor-1}\left(\begin{array}{l}
n \\
i
\end{array}\right)(q-1)^{i}<1 / 2 .
\end{aligned}
$$


Equations (5) and (6) imply that the assumption of Theorem 2 becomes true for sufficiently large $n$, which shows Corollary 3 .

\section{A GV-type existential bound for the stabilizer codes}

Let $C \subset \mathbf{F}_{q}^{2 n}$ be a $\mathbf{F}_{q}$-linear space of dimension $n-k$ self-orthogonal with respect to the standard symplectic inner product in $\mathbf{F}_{q}^{2 n} . C$ can be viewed as an $[[n, k]]_{q}$ stabilizer QECC. Let $A_{n}$ be the set of all such $C$ 's. A nonzero e $\in \mathbf{F}_{q}^{2 n}$ can be viewed as a quantum error on $n$ qudits. Let $A_{n}(\mathbf{e})$ be the set of stabilizer codes that cannot detect e as an error, that is, $A_{n}(\mathbf{e})=\left\{C \in A_{n} \mid \mathbf{e} \in C^{\perp \mathrm{s}} \backslash C\right\}$, where $C^{\perp \mathrm{s}}$ is the dual of $C$ with respect to the symplectic inner product. Then $\sharp A_{n}(\mathbf{e}) \leq \frac{1-q^{-2 k}}{1-q^{-2 n}} \cdot \frac{1}{q^{n-k}} \sharp A_{n}[12$, Lemma 9].

Recall that, for $C$ to be $\left[\left[n, k, d_{x}, d_{z}\right]\right]_{q}, C$ must be able to detect all $d_{x}$ or less bit errors and all $d_{z}$ or less phase errors. The number of such errors is

$$
\sum_{i=1}^{d_{x}-1}\left(\begin{array}{l}
n \\
i
\end{array}\right)(q-1)^{i} \times \sum_{i=1}^{d_{z}-1}\left(\begin{array}{l}
n \\
i
\end{array}\right)(q-1)^{i}
$$

By the same argument as [12, Remark 10] (or as the last section), we have the following theorem:

Theorem 4 Let $n, k_{1}, k_{2}, d_{x}$ and $d_{z}$ be positive integers such that

$$
\frac{1-q^{-2 k}}{1-q^{-2 n}} \cdot \frac{1}{q^{n-k}} \sum_{i=1}^{d_{x}-1}\left(\begin{array}{l}
n \\
i
\end{array}\right)(q-1)^{i} \times \sum_{i=1}^{d_{z}-1}\left(\begin{array}{l}
n \\
i
\end{array}\right)(q-1)^{i}<1
$$

then there exists an $\left[\left[n, k, d_{x}, d_{z}\right]\right]_{q}$ stabilizer $Q E C C$.

By almost the same argument as Corollary 3, we can derive the following asymptotic version of Theorem 4 .

Corollary 5 Let $\delta_{x}$ and $\delta_{z}$ be real numbers such that $0 \leq \delta_{x} \leq 1-1 / q$ and $0 \leq \delta_{z} \leq$ $1-1 / q$. If

$$
h_{q}\left(\delta_{x}\right)+h_{q}\left(\delta_{z}\right)<1-R \leq 1,
$$

then, for sufficiently large $n$, there exists an $\left[\left[n,\lfloor n R\rfloor,\left\lfloor n \delta_{x}\right\rfloor,\left\lfloor n \delta_{z}\right\rfloor\right]\right]_{q}$ stabilizer QECC.

The quantum stabilizer code in Corollary 5 has information rate $R$, relative distance $\delta_{x}$ for bit errors, and relative distance $\delta_{z}$ for phase errors.

By the relation between the CSS and the stabilizer QECCs [3], we see that the assumption in Corollary 3 is less demanding than that in Corollary 5 for the same $n$, $R=R_{1}-R_{2}, \delta_{x}$ and $\delta_{z}$, which means that Corollary 5 is a stronger existential bound than Corollary 3 . 
Remark 6 Theorems 2 and 4 and Corollaries 3 and 5 do not admit direct comparisons against previously known GV-type bounds even when $d_{x}=d_{z}$. The reason is as follows: For a binary QECC to be $[[n, k, 2,2]]_{2}$, it must detect at least $n^{2}$ different errors. On the other hand, for a binary $[[n, k]]_{2}$ QECC to detect all single symmetric errors, it only has to detect $3 n$ errors, which is generally much fewer than $n^{2}$. The above example shows that the number of asymmetric quantum errors is much different from that of corresponding symmetric quantum errors, even if we assume the same number of bit errors and phase errors in asymmetric quantum errors.

In addition, the famous $[[5,1,3]]_{2}$ binary stabilizer code in $[3,8]$ can detect up to four bit errors if there is no phase error, and can detect up to four phase errors if there is no bit error. Thus, it is simultaneously both $[[5,1,1,5]]_{2}$ AQECC and $[[5,1,5,1]]_{2}$ AQECC. This phenomenon makes the direct comparison even more difficult.

Acknowledgements The author would like to thank an anonymous reviewer for careful reading and the helpful report that improved this note, and Prof. Fernando Hernando for drawing his attention to the asymmetric quantum error correction.

Open Access This article is distributed under the terms of the Creative Commons Attribution 4.0 International License (http://creativecommons.org/licenses/by/4.0/), which permits unrestricted use, distribution, and reproduction in any medium, provided you give appropriate credit to the original author(s) and the source, provide a link to the Creative Commons license, and indicate if changes were made.

\section{References}

1. Ashikhmin, A., Knill, E.: Nonbinary quantum stabilizer codes. IEEE Trans. Inform. Theory 47(7), 3065-3072 (2001)

2. Calderbank, A.R., Rains, E.M., Shor, P.W., Sloane, N.J.A.: Quantum error correction and orthogonal geometry. Phys. Rev. Lett. 78(3), 405-408 (1997)

3. Calderbank, A.R., Rains, E.M., Shor, P.W., Sloane, N.J.A.: Quantum error correction via codes over GF(4). IEEE Trans. Inform. Theory 44(4), 1369-1387 (1998)

4. Calderbank, A.R., Shor, P.W.: Good quantum error-correcting codes exist. Phys. Rev. A 54(2), 10981105 (1996)

5. Ekert, A., Macchiavello, C.: Quantum error correction for communication. Phys. Rev. Lett. 77(12), 2585-2588 (1996)

6. Feng, K., Ma, Z.: A finite Gilbert-Varshamov bound for pure stabilizer quantum codes. IEEE Trans. Inform. Theory 50(12), 3323-3325 (2004). doi:10.1109/TIT.2004.838088

7. Galindo, C., Geil, O., Hernando, F., Ruano, D.: Improved constructions of nested code pairs. IEEE Trans. Inform. Theory (2017). doi:10.1109/TIT.2017.2755682

8. Gottesman, D.: Class of quantum error-correcting codes saturating the quantum Hamming bound. Phys. Rev. A 54(3), 1862-1868 (1996)

9. Ioffe, L., Mézard, M.: Asymmetric quantum error-correcting codes. Phys. Rev. A 75, 032345 (2007). doi:10.1103/PhysRevA.75.032345

10. Jin, L., Xing, C.: Quantum Gilbert-Varshamov bound through symplectic self-orthogonal codes. In: Proceedings 2011 IEEE International Symposium on Information Theory, pp. 455-458. Sait Petersburg, Russia (2011). doi:10.1109/ISIT.2011.6034167

11. MacWilliams, F.J., Sloane, N.J.A.: The Theory of Error-Correcting Codes. Elsevier, Amsterdam (1977)

12. Matsumoto, R., Uyematsu, T.: Lower bound for the quantum capacity of a discrete memoryless quantum channel. Jo. Math. Phys. 43(9), 4391-4403 (2002). doi:10.1063/1.1497999

13. Nielsen, M.A., Chuang, I.L.: Quantum Computation and Quantum Information. Cambridge University Press, Cambridge (2000)

14. Sarvepalli, P.K., Klappenecker, A., Rötteler, M.: Asymmetric quantum codes: constructions, bounds and performance. Proc. R. Soc. A Math. Phys. Eng. Sci. 465(2105), 1645-1672 (2009). doi:10.1098/ rspa.2008.0439 
15. Steane, A.M.: Multiple particle interference and quantum error correction. Proc. R. Soc. Lond. Ser. A 452(1954), 2551-2577 (1996)

16. Steane, A.M.: Simple quantum error-correcting codes. Phys. Rev. A 54(6), 4741-4751 (1996). doi:10. 1103/PhysRevA.54.4741 